\title{
Temporal shaping and pulse-compression in thermography using laser heating
}

\author{
by P. Hirsch*, H. Malekmohammadi**, S. Ahmadi*, C. Hassenstein*, N. W. Pech-May*, S. Laureti***, \\ M. Ricci***, M. Ziegler*

\begin{abstract}
* Bundesanstalt für Materialforschung und -prüfung (BAM), 12200 Berlin, Germany, philipp-daniel.hirsch@bam.de ** Department of Engineering, University of Perugia, Polo Scientifico Didattico di Terni, 05100 Terni, Italy *** Department of Informatics, Modeling, Electronics and System Engineering, The University of Calabria, 87036 Rende, Italy
\end{abstract}

\begin{abstract}
The shaping of the heating radiation in the time domain and the corresponding signal processing, i.e. Pulsecompression Thermography, show promises in combining the advantages of both pulse and lock-in thermography. At the same time, the inherent disadvantage of the often-used thermal-based light sources can be addressed using planar illuminating high-power lasers. Prospectively, pulse-compression can thus become a new modality in infrared thermographic inspection.
\end{abstract}

\section{Introduction}

One of the main tasks of non-destructive testing is to verify the internal structure of solids, e.g. the presence of cracks and voids. One solution is active thermography, in which the diffusion of heat flow is disturbed by inhomogeneities. In pulsed thermography [1, 2] heat flow is generated by pulsed excitation, such as a flash lamp. For optimal detection, a very short pulse with high power is required to obtain a good signal-to-noise ratio (SNR) and a better depth range. However, excessive power can damage the tested specimen' surface. To avoid such a problem, periodic thermal wave lock-in thermography $[3,4,5,6]$ which is based on periodic excitation provided by e.g. halogen lamps, can be used to probe different depths with similar SNR. However, long periodic excitations are required for a good SNR at low power levels.

Similar to the RADAR or ultrasound applications, an alternative solution is the temporal shaping of a pulsed excitation. This is showed to combine the advantages of both mentioned approaches, such as a lower required power, an sufficient SNR and a short measurement time. The use of a frequency or phase modulated heating stimulus and its subsequent cross-correlation with the recorded thermograms leads to the pulse-compression (PuC) technique, which is a mixed form of the two presented thermographic methods [7].

Therefore, both higher depth ranges and depth resolutions can be achieved compared to the classical methods of mono-frequency lock-in and high-energy short pulsed excitation, but so far only marginal improvements of the SNR compared to conventional flash thermography were possible. The reason is probably the low irradiance (significantly lower than flash lamps) and low modulation bandwidths of the halogen lamps used [7]. Even if lasers were used, there were only pulsed lasers and only very small areas of a few $\mathrm{mm}^{2}$ were examined [8].

In this work, we try to remove this limitation and analyze the full potential of the temporal shaping and PuC approach in combination with laser heating source. The idea is to use modulation functions having the highest possible autocorrelation and frequencies defined for the application. In contrast to flash thermography, it is therefore possible to adapt the frequency components to the requirements and to transfer more energy over a longer period of time.

The application of chirp signals and optimizations techniques have been already implemented and investigated by many authors, see for example [9], but binary sequences possible enable higher autocorrelation, lower sidelobe level and hence better SNR compared to the chirp signals. Among binary sequences, Barker code and pseudo-noise binary sequences such as Maximum Length Sequences and Legendre sequences are most often used in non-destructive testing applications $[10,11,12,13]$. Furthermore, the advantage of high-power lasers and the application of PuC will be presented.

\section{Active thermography using high-power laser arrays}

For a material with thermal conductivity $k$, emissivity $\varepsilon$ or absorption coefficient $a$, density $\rho$ and specific heat capacity $c_{p}$, the total radiant power emitted from a surface $F$ that was previously at room temperature $T_{0}$, by applying a short irradiation of $\Delta t$ with the irradiance $q$ after time $t$, can be obtained as follows:

$$
P_{\varepsilon}=\varepsilon \sigma F\left(T_{0}+\frac{a q \Delta t}{\sqrt{\rho c_{p} k \pi t}}\right)^{4}+(1-\varepsilon) P_{\text {source }}
$$

where $\sigma$ is the Stefan-Boltzmann constant and $P_{\text {source }}$ is the radiant power of the source in the detection range of the thermal camera. This results in the limitation of the useful signal (second term in brackets) to the ambient radiation (first term in brackets) and the interfering radiation of the source (last summand), especially for small $\varepsilon, a$ and large $\rho, c_{p}, k$. Therefore, the use of a proper source is essential. 
Halogen lamps are mainly used due to their low investment and operating costs. They have disadvantages such as their very broad emission spectrum and their small modulation bandwidth. They can practically only be electrically modulated between continuous wave operation and approx. $1 \mathrm{~Hz}$. These disadvantages are addressed using LEDs and lasers as heating sources, which has been investigated in recent years. Electroluminescence (with diode lasers) generates hardly any heat radiation and thus enables testing during the heating process with spectral separation of the light source from the detector. These sources are intrinsically fast, and their modulation bandwidth is practically limited to about $1 \mathrm{MHz}$ only by the available control devices. A new laser-based heat source is the vertical-cavity surface-emitting laser (VCSEL) array, which has been characterized for thermographic non-destructive testing and validated for use in high-frequency lock-in thermography [14]. Furthermore, this source has proven to be suitable for novel interference methods with thermal waves [15] and for super resolution thermography [16]. The mentioned laser source is used here together with PuC and pulse excitation for the comparative analysis of CFRP samples.

\section{Application of pulse-compression on laser-based heat stimulation}

In order to prove the proposed scheme, a quasi-isotropic carbon fiber reinforced polymer (CFRP) plate with real defect (an impact damage of $6 \mathrm{~J}$ ) was chosen and tested. The excitation signal was a pseudo-noise binary sequence of two different lengths $L=53$ and 103. Each sequence was consisting of a train of square pulses in time domain with minimum pulse-length $T_{\text {bit }}=0.02 \mathrm{~s}$ and $0.04 \mathrm{~s}$. Selection of $T_{\text {bit }}$ defines the desired bandwidth of the signal in frequency domain. The excitation signal then was fed into the VCSEL system through an arbitrary waveform generator, illuminating the sample's frontside, and the thermal signal was recorded by the infrared camera with proper synchronization at the frame rate of 500 $\mathrm{Hz}$ in reflection mode.

In the second test scheme, instead of a binary sequence, a single laser pulse with a pulse duration equal to $T_{b i t}$ but with higher power was used to maintain the same maximum surface temperature.

Fig. 1 shows the results of the two test schemes. The parts (a) and (b) show the results (best frames) of the analysis of cooling stage for the single pulse laser in frequency domain, while (c) and (d) show the results (best frames) for the corresponding binary sequence of the same $T_{b i t}$ after applying PuC. It can be observed that the PuC scheme shows similar detection capability with significantly lower peak power.
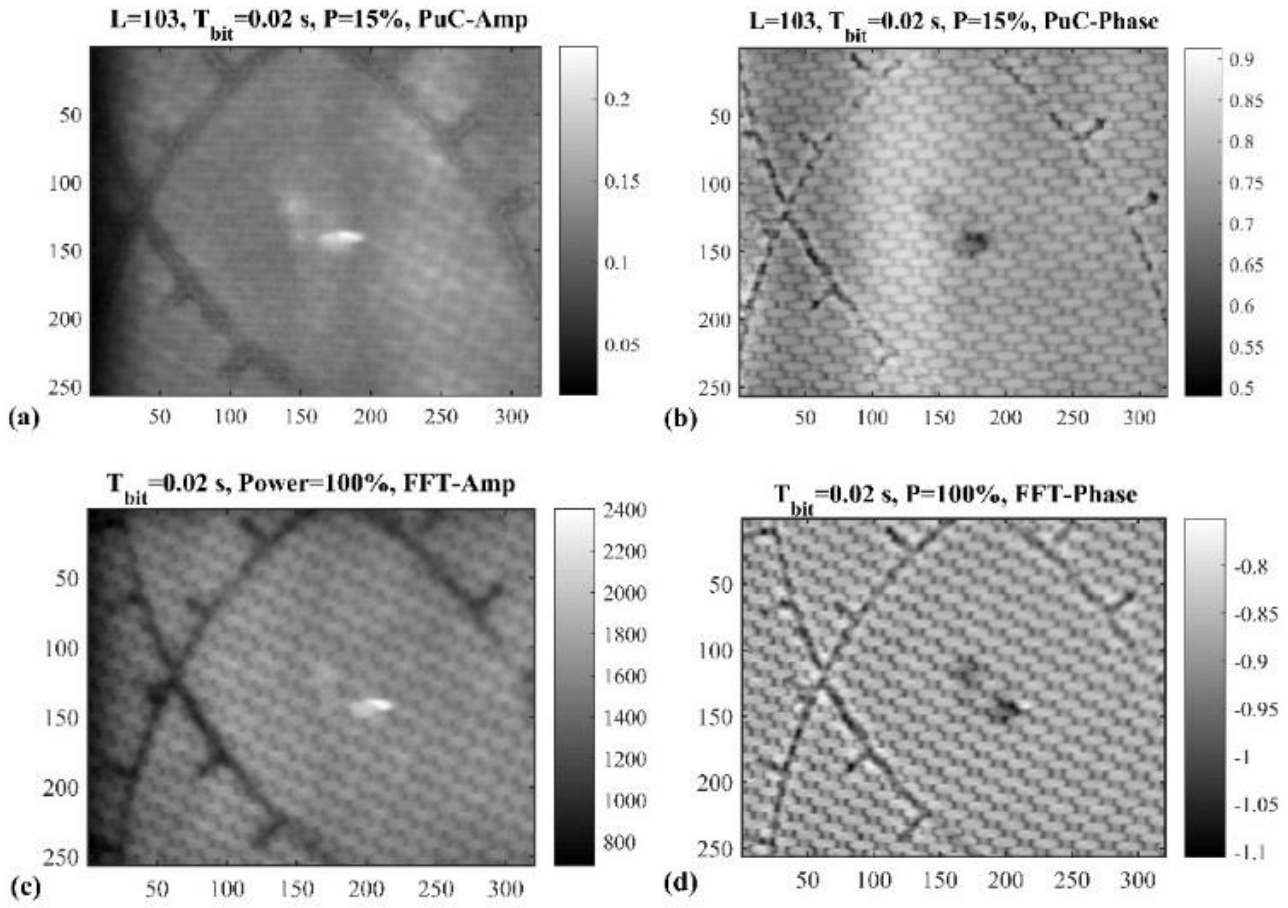

Fig. 1. Experimental results for two test schemes: (a) and (b) PuC amplitude and phase images respectively for Legendre sequence of $L=103, T_{b i t}=0.02 s$ and peak power of $15 \%$, (c) and (d) FFT amplitude and phase respectively for a single laser pulse of duration $T=0.02 \mathrm{~s}$ and peak power of $100 \%$

\section{Acknowledgement}

Gefördert durch die Deutsche Forschungsgemeinschaft (DFG) - 400857558, funded by the Deutsche Forschungsgemein-schaft (DFG, German Research Foundation) - 400857558. This research work was partially supported by the European Union's Horizon 2020 research and innovation programme under the Marie Skłodowska-Curie grant agreement No 722134-NDTonAIR 


\section{REFERENCES}

[1] ALMOND, D. P.; PICKERING, S. G.; J. Appl. Phys. 111, 093510, 2012.

[2] MALDAGUE, X.; MARINETTI, S.; J. Appl. Phys. 79, 2694, 1996.

[3] CARLOMAGNO, G. M. et al.; Proc. 3rd Biannual Inform. Exchange, St. Louis/USA,33-39, 1976.

[4] BEAUDOIN, J. L. et al.: Infrared Techn. \& Appl.. SPIE 590,287, 1985.

[5] KUO, P. K.; FENG, Z. J.; AHMED, T.; FAVRO, L. D.; THOMAS, R. L.; HARTIKAINEN, J.; Photoacoustic and photothermal phenomena, Springer-Verlag, Heidelberg (1988), S. 415-418

[6] BUSSE, G.; WU, D.; KARPEN, W.: Thermal wave imaging with phase sensitive modulated thermography. J. Appl. Phys. 718 (1992), S. 3962-3965

[7] MULAVEESALA, R.; TULI, S.; Appl. Phys. Lett. 89(19),191913, 2006.

[8] KAIPLAVIL, S.; MANDELIS, A.; Nature Photonics, 8(8), 635, 2014.

[9] Malekmohammadi, $\mathrm{H}$. et al., Comparison of optimization strategies for the improvement of depth detection capability of Pulse Compression Thermography, Quantitative Infrared Thermography Journal, 1-14, 2019

[10] Yi, Q., et al., Quantitative evaluation of crack depth on a thin aluminum plate by using eddy current pulsecompression thermography, IEEE Transactions on Industrial Informatics, 2019

[11] Hutchins, D., et al., Coded waveforms for optimised air-coupled ultrasonic non-destructive evaluation, Ultrasonics, Vol. 54, pp. 1745-1759, 2014

[12] Zhang, G; Zhou, Q. Pseudonoise codes constructed by Legendre sequence. Electronics Letters. 2002, 38(8), 577 376-377

[13] Ghali, V., et al., Barker coded thermal wave imaging for defect detection in carbpn fiber reinforced plastics, Insight Non-Destructive Testing and Condition Monitoring, vol. 53, no. 11, pp. 621-624, 2011

[14] M. Ziegler, E. Thiel, T. Studemund, Materials Testing: Vol. 60, No. 7-8, pp. 749-757, 2018.

[15] E. Thiel, M. Ziegler, T. Studemund, Int J Thermophys 40, 17, 2019.

[16] P. Burgholzer, T. Berer, M. Ziegler, E. Thiel, S. Ahmadi, J. Gruber, G. Mayr \& G. Hendorfer, Quantitative InfraRed Thermography Journal, DOI: 10.1080/17686733.2019.1655247, 2019. 